\title{
Low expression of NCALD is associated with chemotherapy resistance and poor prognosis in epithelial ovarian cancer
}

\author{
Li-yuan Feng and Li Li
}

\begin{abstract}
Background: Low expression of NCALD(neurocalcin delta) in peripheral blood of ovarian cancer patients predicts poor prognosis. However, the molecular mechanism of NCALD in ovarian cancer and its relationship with chemotherapy outcomes is unclear. The aim of this study was to investigate the potential signaling pathways of NCALD and to evaluate its ability to predict chemotherapy outcomes and prognosis.

Methods: High-throughput RNA sequencing data were downloaded from TCGA. GSEA explored the potential signaling pathways of NCALD. The expression of NCALD in chemotherapy sensitive and chemotherapy resistant ovarian cancer patients was detected by TCGA data and clinical samples. ROC analysis confirmed the ability of NCALD to predict chemotherapy outcomes. The association between NCALD expression and prognosis in ovarian cancer patients was assessed using Kaplan-Meier plotter.

Results: In patients with NCALD overexpression, genes expression related to ERK1 / 2 signaling pathway, NF-kappaB signaling pathway, TGF- $\beta$ signaling pathway and immune response pathway was increased, especially ERK1 / 2 signaling pathway. The expression of NCALD in chemoresistant patients was significantly lower than chemosensitive patients. In TCGA data and immunohistochemical samples, the AUC of NCALD expression predicting chemotherapy outcome was 0.59 and 0.64, respectively. In clinical samples, low expression of NCALD was associated with poor OS and PFS.

Conclusions: NCALD may activate the ERK1 / 2 signaling pathway in ovarian cancer. As a new biomarker of chemotherapy sensitivity, NCALD was significantly down-regulated in chemotherapy resistance ovarian cancer patients. Low expression of NCALD in ovarian cancer is associated with poor OS and PFS. In the future, further research will be needed on the potential mechanism and clinical application value of NCALD in ovarian cancer.
\end{abstract}

Keywords: Ovarian cancer, Chemoresistance, NCALD, Prognosis

\section{Background}

Ovarian cancer is the most lethal gynecological malignancy. More than $75 \%$ of ovarian cancer patients are stage III or IV at the time of initial diagnosis, with a 5-year survival rate was less than $30 \%[1,2]$. The standard treatment plan for the initial diagnosis of ovarian cancer is optimal debulking combined with platinum-based chemotherapy

\footnotetext{
* Correspondence: lili@gxmu.edu.cn

Department of Gynecologic oncology, Guangxi Medical University Cancer Hospital, 71 Hedi Road, Nanning, Guangxi 530021, P.R. China
}

[3]. Recent advances in radical surgery and chemotherapy have improved the treatment outcomes for ovarian cancer. However, chemotherapy resistance in the later stage of treatment results in high recurrence, metastasis and mortality $[4,5]$. Current prognostic indicators including age, stage, and histological type are insufficient to predict the efficacy of conventional chemotherapy [6, 7]. In order to improve the prognosis of ovarian cancer patients, new biomarkers which predict chemotherapy resistance and treatment strategies to overcome resistance are needed.

(C) The Author(s). 2020 Open Access This article is licensed under a Creative Commons Attribution 4.0 International License, which permits use, sharing, adaptation, distribution and reproduction in any medium or format, as long as you give appropriate credit to the original author(s) and the source, provide a link to the Creative Commons licence, and indicate if changes were made. The images or other third party material in this article are included in the article's Creative Commons licence, unless indicated otherwise in a credit line to the material. If material is not included in the article's Creative Commons licence and your intended use is not permitted by statutory regulation or exceeds the permitted use, you will need to obtain permission directly from the copyright holder. To view a copy of this licence, visit http://creativecommons.org/licenses/by/4.0/ The Creative Commons Public Domain Dedication waiver (http://creativecommons.org/publicdomain/zero/1.0/) applies to the data made available in this article, unless otherwise stated in a credit line to the data. 
NCALD(neurocalcin delta) is a member of the neuronal calcium sensor family, which is involved in calcium signaling pathway and G-protein-coupled receptor signaling pathway. It is abundant in normal brain, testis, ovary and small intestine $[8,9]$. NCALD consists of four EF hand motifs, but only three can bind to calcium, which alters the conformation of the NCALD protein [10]. Studies have found that NCALD expression is associated with the prognosis of acute myeloid leukemia and non-small cell lung cancer, indicating its clinical potential as a prognosis biomarker [11, 12]. In ovarian cancer, Isaksson's study showed that low expression of NCALD predicts poor prognosis [13]. Since Isaksson's NACLD data are from peripheral blood. It would be important to look for the association of NACLD expression with PFS and OS to strengthen the findings. In addition, the molecular mechanism of NCALD in ovarian cancer and its relationship with chemotherapy sensitivity has not been reported. The aim of our study was to investigate the potential signaling pathways of NCALD and to evaluate its ability to predict chemotherapy outcomes and prognosis.

\section{Materials and methods TCGA data}

NCALD mRNA expression (data_mRNA_median_Zscores) and clinical data of ovarian cancer in TCGA database downloaded from cbioportal (http://www.cbioportal.org/). Ovarian cancer patients without chemotherapy outcomes were excluded. Ovarian cancer patients with gender, age, grade, FIGO stage, survival outcome, chemotherapy outcome and NCALD expression were included.

\section{GSEA explored the potential signal pathway of NCALD in ovarian cancer}

Ovarian cancer samples in TCGA were divided into low expression group and high expression group according to the NCALD expression mean. Gene enrichment analysis was performed using GSEA software (https://www. broadinstitute.org/gsea/). Number of permutation = 2500, gene sets with $P$ values $<0.005$ and FDR q values $<0.20$ are considered to be significantly enriched gene sets.

\section{Patient samples}

From March 1997 to April 2013, 178 epithelial ovarian cancer patients were collected from the Guangxi Medical University Cancer Hospital. One hundred seventyeight paraffin tissues were used for immunohistochemistry, and 61 frozen tissues were used for QRT-PCR. The inclusion and exclusion criteria are as follows. Inclusion criteria: (1) patients with chemotherapy outcomes; (2) patients with postoperative pathology; (3) patients with survival outcomes; and (4) patients with complete clinical data (including gender, age, grade and FIGO stage). Exclusion criteria: (1) patients without definite postoperative pathology; (2) patients without complete data

Table 1 Clinical characteristics of patients

\begin{tabular}{|c|c|c|c|c|c|c|c|c|}
\hline \multirow[b]{2}{*}{$\begin{array}{l}\text { Clinical } \\
\text { characteristics }\end{array}$} & \multicolumn{4}{|l|}{ TCGA(N $=287)$} & \multicolumn{4}{|l|}{$\mathrm{IHC}(N=178)$} \\
\hline & All (287) & chemoresistant (90) & chemosensitive (197) & $P$ & All (178) & chemoresistant (71) & chemosensitive (107) & $P$ \\
\hline \multicolumn{9}{|l|}{ FIGO stage } \\
\hline$|-| \mid$ & $14(4.88 \%)$ & $1(1.11 \%)$ & $13(6.60 \%)$ & 0.088 & $38(22.09 \%)$ & $8(11.76 \%)$ & $30(28.85 \%)$ & $0.008^{*}$ \\
\hline III-IV & $273(95.12 \%)$ & 89 (98.89\%) & 184 (93.40\%) & & $134(77.91 \%)$ & 60 (88.24\%) & $74(71.15 \%)$ & \\
\hline Missing & 0 & 0 & 0 & & 6 & 3 & 3 & \\
\hline \multicolumn{9}{|l|}{ Grade } \\
\hline 3 & $243(86.48 \%)$ & 81 (91.01\%) & $162(84.38 \%)$ & 0.130 & $141(86.50 \%)$ & $57(86.36 \%)$ & $84(86.60 \%)$ & 0.989 \\
\hline 2 & $38(13.52 \%)$ & 8 (8.99\%) & $30(15.62 \%)$ & & $16(9.82 \%)$ & $7(10.61 \%)$ & $9(9.28 \%)$ & \\
\hline 1 & 0 & 0 & 0 & & $6(3.68 \%)$ & $2(3.03 \%)$ & $4(4.12 \%)$ & \\
\hline Missing & 6 & 1 & 5 & & 15 & 5 & 10 & \\
\hline \multicolumn{9}{|l|}{ Histology types } \\
\hline Serous & 287 & 90 (100.00\%) & 197 (100.00\%) & - & $129(72.47 \%)$ & 46 (64.79\%) & $83(77.57 \%)$ & 0.078 \\
\hline Mucinous & 0 & $0(00.00 \%)$ & $0(00.00 \%)$ & & $10(5.62 \%)$ & $6(8.45 \%)$ & $4(3.74 \%)$ & \\
\hline Others & 0 & $0(00.00 \%)$ & $0(00.00 \%)$ & & 39 (21.91\%) & 19 (26.76\%) & $20(18.69 \%)$ & \\
\hline \multicolumn{9}{|c|}{ Surgical debulking } \\
\hline Optimal & $56(21.54 \%)$ & $9(10.59 \%)$ & $47(26.86 \%)$ & $0.003^{*}$ & $71(64.55 \%)$ & $28(53.85 \%)$ & $43(74.14 \%)$ & $0.026^{*}$ \\
\hline Suboptimal & $204(78.46 \%)$ & 76 (89.41\%) & $128(73.14 \%)$ & & 39 (35.45\%) & 24 (46.15\%) & 15 (25.86\%) & \\
\hline Missing & 27 & 5 & 22 & & 68 & 19 & 49 & \\
\hline
\end{tabular}

Note: $p$ value was compared between the chemosensitive patients and the chemoresistant patients, "** means $p<0.05$ 
(including chemotherapy outcomes, survival outcomes and clinical data). All Samples received the patient's informed consent and the approval of the Ethics Committee of the Guangxi Medical University Cancer Hospital.

Chemoresistance was defined as the patients who do not achieve a complete response after initial treatment, or with relapse within 6 months after complete response. Chemosensitivity was defined as patients with recurrence more than 6 months after complete response. Overall survival (OS) was defined as the time from diagnosis to death due to ovarian cancer. Progressionfree survival (PFS) was defined as the time from initial treatment to tumor progression.

\section{RNA extraction and QRT-PCR}

RNA extraction and reverse transcription were performed according to the Genomic RNA Purification Kit (Thermo Scientific, Cat. No. K0731) and Revert Aid First Strand cDNA Synthesis Kit (Thermo Scientific, Cat. No. K1622) instructions. One Step TB Green ${ }^{\circ}$ PrimeScriptTM RT-

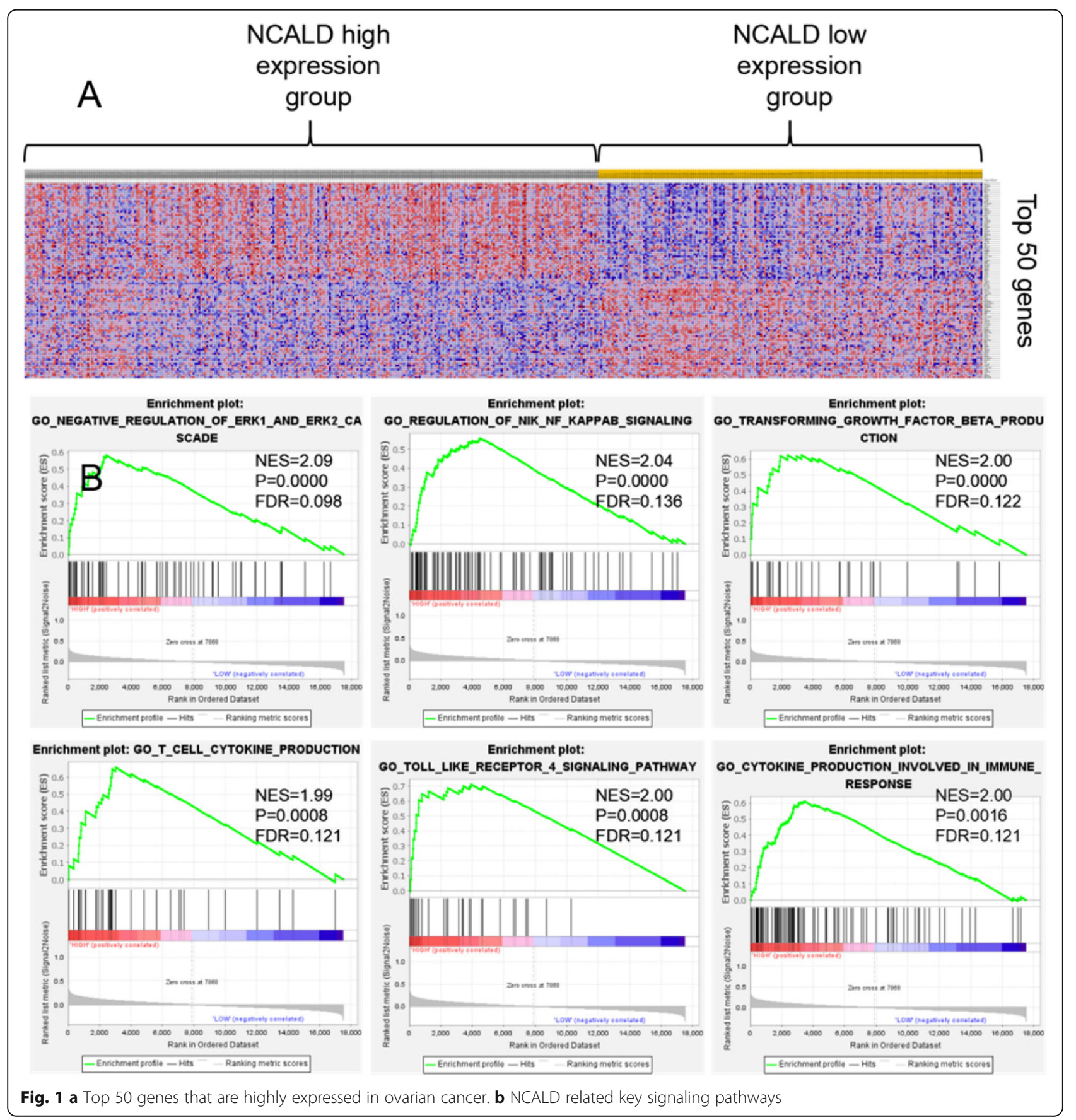


PCR Kit (Takara, Cat. No. RR066B) was used for real-time PCR on an ABI step-one plus PCR machine.

\section{Tissue microarray and immunohistochemistry}

The pathological types were confirmed by HE stain. Each tumor has 2-3 repeated tissue spots. The diameter is $1 \mathrm{~mm}$. Immunohistochemistry was performed according to the ready-to-use immunohistochemical ultrasensitive UltraSensitiveTM SP test kit (maixin, Cat. No. KIT9710) instructions. NCALD concentration is 1:400 (Abcam, Cat. No.ab155161). Two pathologists read the pathological sections independently. The score criteria are as follows: Positive cell ratios of $<1,1-25 \%, 25-50 \%$, $50-75 \%$ and $75-100 \%$ were assigned $0,1,2,3,4$ points, respectively. Stain intensity of no coloring, light yellow, yellow, brown were assigned $0,1,2,3$ points, respectively. The product of positive cell ratio and stain intensity is stain index. Stain index $\leq 6$ points was classified as low expression, while $>6$ points was classified as high expression [14].

\section{Statistical analysis}

SPSS17.0 was used for analysis. T test (measurement data) and chi-square test (categorical data) were used for comparison between the two groups. ROC analysis confirmed the ability of NCALD to predict chemotherapy outcomes. The association between NCALD expression and prognosis was assessed using Kaplan-Meier plotter.

The association between NCALD expression and clinicopathological parameters was assessed with Spearman test. $P$ values were two-sided, and $P<0.05$ was considered statistically significant.

\section{Results}

\section{Characteristics of patients}

Data from 491 ovarian cancer patients were downloaded from the TCGA, and 204 patients without chemotherapy outcomes were excluded. 287 ovarian cancer patients with gender, age, grade, FIGO stage, survival outcome, chemotherapy outcome, and NCALD mRNA expression were obtained. The clinical characteristics are shown in Table 1. Among the 178 clinical samples, 34 had FIGO stage I or II and 134 patients had FIGO stage III or IV. There were 141 patients with grade 3 and 22 patients with grade $1-2$. There were 129 patients with serous type and 49 patients with other histology types.

Optimal surgical debulking rate was $21.54 \%(56 / 260)$ and $64.55 \%$ (71/110) in TCGA data and clinical samples, respectively. In TCGA and clinical samples, the number of patients with chemotherapy resistance who achieved optimal surgical debulking was significantly less than patients with chemotherapy sensitivity (TCGA: 9 vs 47, clinical samples: 28 vs 43). In clinical samples, the number of chemotherapy resistant patients with stage III-IV was significantly higher than stage I-II ( 8 vs 30$)$, but there was no difference in TCGA data.

\section{Potential signal pathways of NCALD in ovarian cancer}

Four hundred ninety-one Ovarian cancer samples in TCGA were divided into low expression group and high expression group according to the NCALD expression mean. The transcriptome data between 294 patients with NCALD high-expression and 197 patients with NCALD low-expression was compared. The key cellular processes and signal pathways of NCALD in ovarian cancer were identified by gene set enrichment analysis (GSEA). There were 17,518 differentially expressed genes in the two groups, 7868 (44.90\%) genes were highly expressed in the NCALD high-expression group, and 9650 $(55.10 \%)$ genes were highly expressed in the NCALD low-expression group. The complete list of differentially expressed genes is shown in supplementary document 1. The heat map shows the top 50 genes that are highly expressed in the NCALD overexpression group and the low-expression group, respectively, see Fig. 1a.

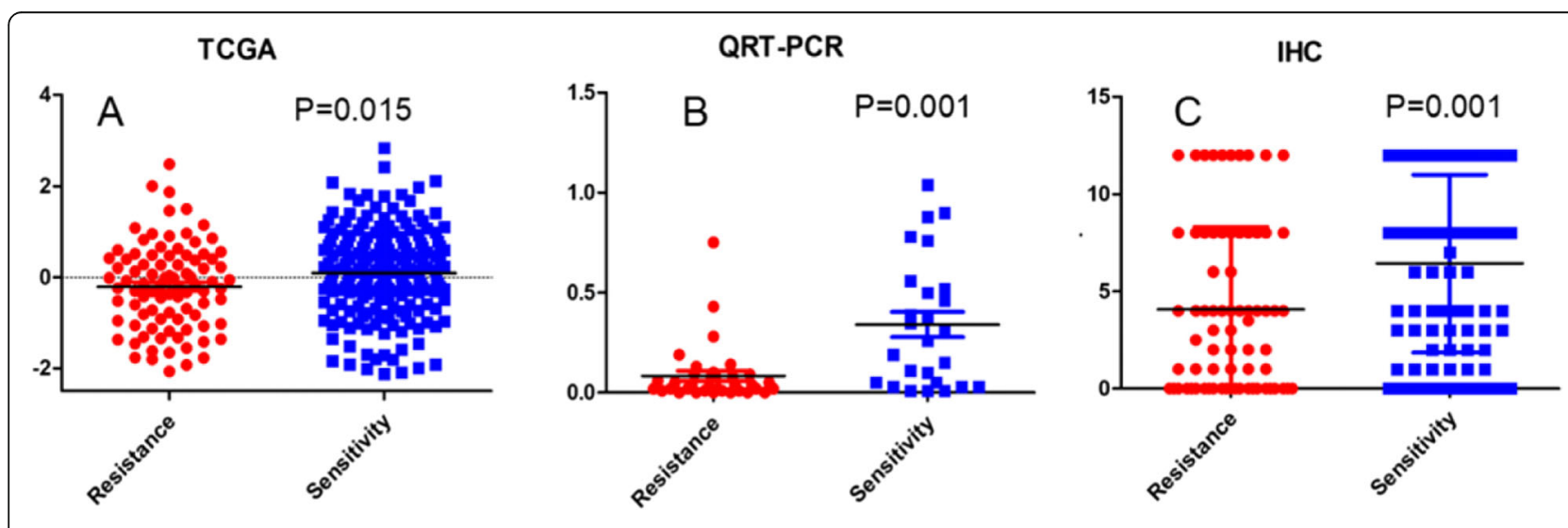

Fig. 2 a Expression of NCALD in TCGA data. b Expression of NCALD in clinical samples (QRT-PCR). c Expression of NCALD in clinical samples (IHC) 


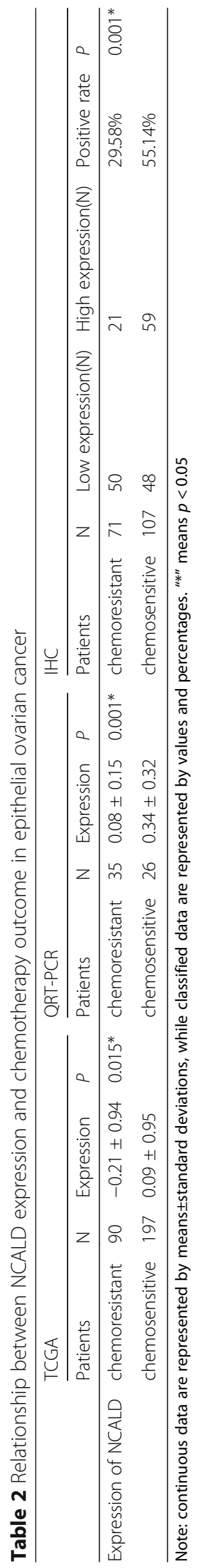


Gene set enrichment analysis (GSEA) showed that the genes expression related to ERK1/2 signal pathway, NFkappaB signal pathway, TGF- $\beta$ signal pathway, and immune response pathway was increased in the NCALD overexpression group, especially ERK1 / 2 signaling pathway $(\mathrm{NES}=2.09)$. See Fig. $1 \mathrm{~b}$. The complete list of differential gene set is shown in supplementary document 2 .

\section{Expression of NCALD in chemosensitive and}

\section{chemoresistant ovarian cancerpatients in TCGA data}

The expression of NCALD in chemoresistant patients was significantly lower than that in chemosensitive patients. The mean \pm standard deviation was $-0.21 \pm 0.94$ VS $0.09 \pm 0.95$, see Fig. 2a. Spearman correlation analysis showed no correlation between NCALD expression and FIGO stage, grade and residual disease. Data are not shown.

\section{Expression of NCALD in chemosensitive and} chemoresistant ovarian cancer patients in clinical samples Clinical samples were used to further verify the expression of NCALD in chemosensitive and chemoresistant ovarian cancer patients. QRT-PCR results showed that the expression of NCALD in chemoresistant ovarian cancer patients was significantly lower than that in chemosensitive ovarian cancer patients $(0.08 \pm 0.15$ VS $0.34 \pm 0.32$ ), see Fig. $2 b$.

Immunohistochemistry results showed that NCALD is mainly expressed in the cytoplasm, and a small amount is expressed in the cell membrane. NCALD was highly expressed in 59/107(55.14\%) chemosensitive patients and 21/71(29.58\%) chemoresistant patients. A chi-square test showed that the protein expression of NCALD in chemoresistant patients was significantly reduced compared with chemosensitive patients, as showed in Table 2 and Fig. 2c. Immunohistochemical stain is shown in Fig. 3.

\section{Stratified analysis}

In clinical samples, stratified analysis results showed that in FIGO III-IV stage patients, poorly differentiated patients and other epithelial histology types (except serous and mucinous) patients, NCALD expression in chemotherapy sensitive patients was higher than that in chemotherapy resistant patients $(54.05 \%$ VS $30.00 \%, P=0.005 ; 59.00 \%$ VS $33.33 \%, P=0.002 ; 35.00 \%$ VS $00.00 \%, P=0.015$ ). There was no significant difference in the stratified analysis of the TCGA data. See supplementary document 3.

\section{The ability of NCALD to predict chemotherapy outcomes} In TCGA data and clinical samples, ROC analysis showed that the sensitivity, specificity, accuracy and AUC of NCALD expression to predict chemotherapy sensitivity were $0.70,0.46,0.53,0.59(95 \% \mathrm{CI} 0.52-0.66)$ and $0.59,0.68,0.61,0.64(95 \% \mathrm{CI} 0.56-0.73)$ respectively. The ROC curve was shown in Fig. 4a and b. The positive likelihood ratio and negative likelihood ratio was shown in Table 3.

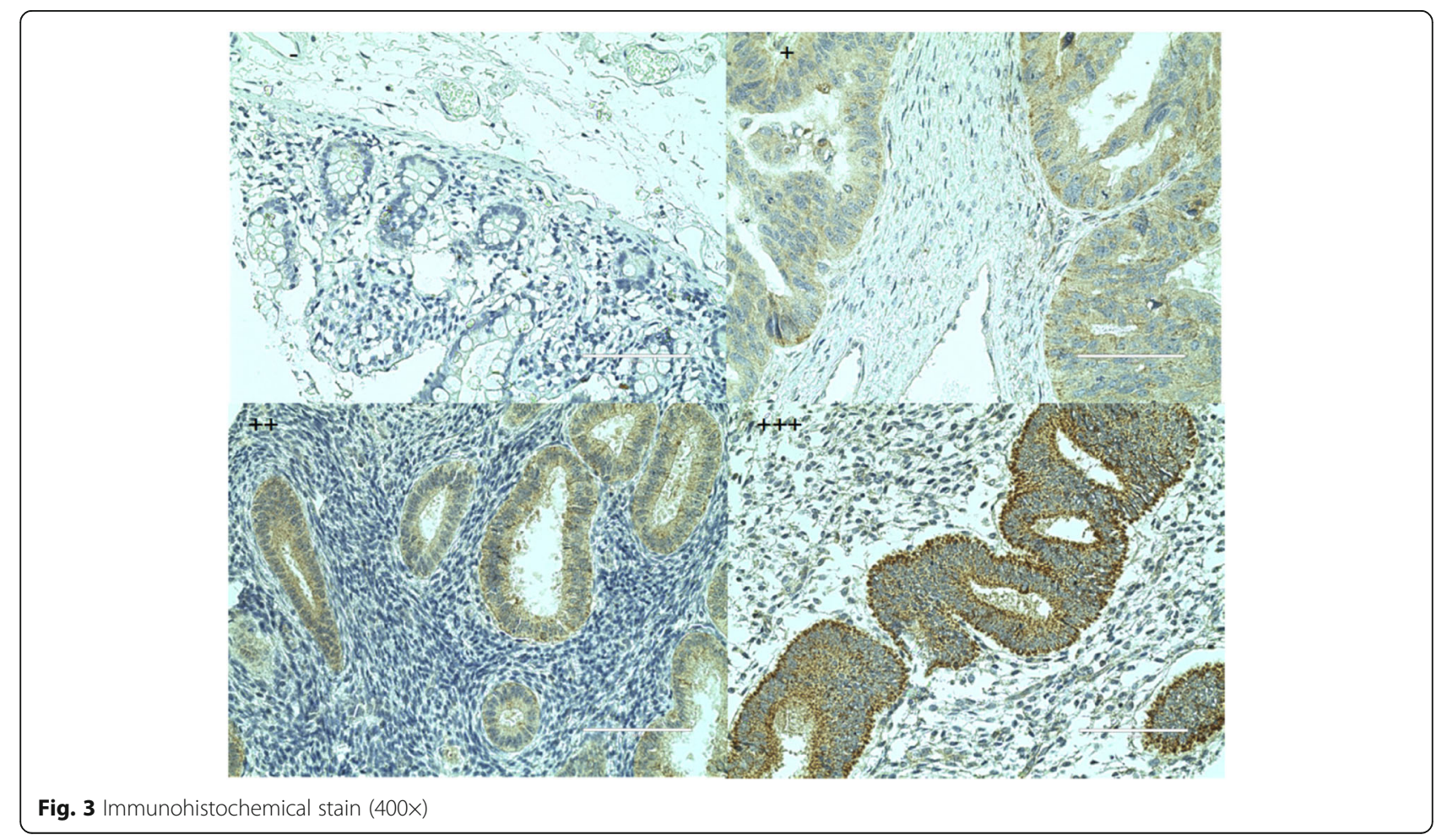




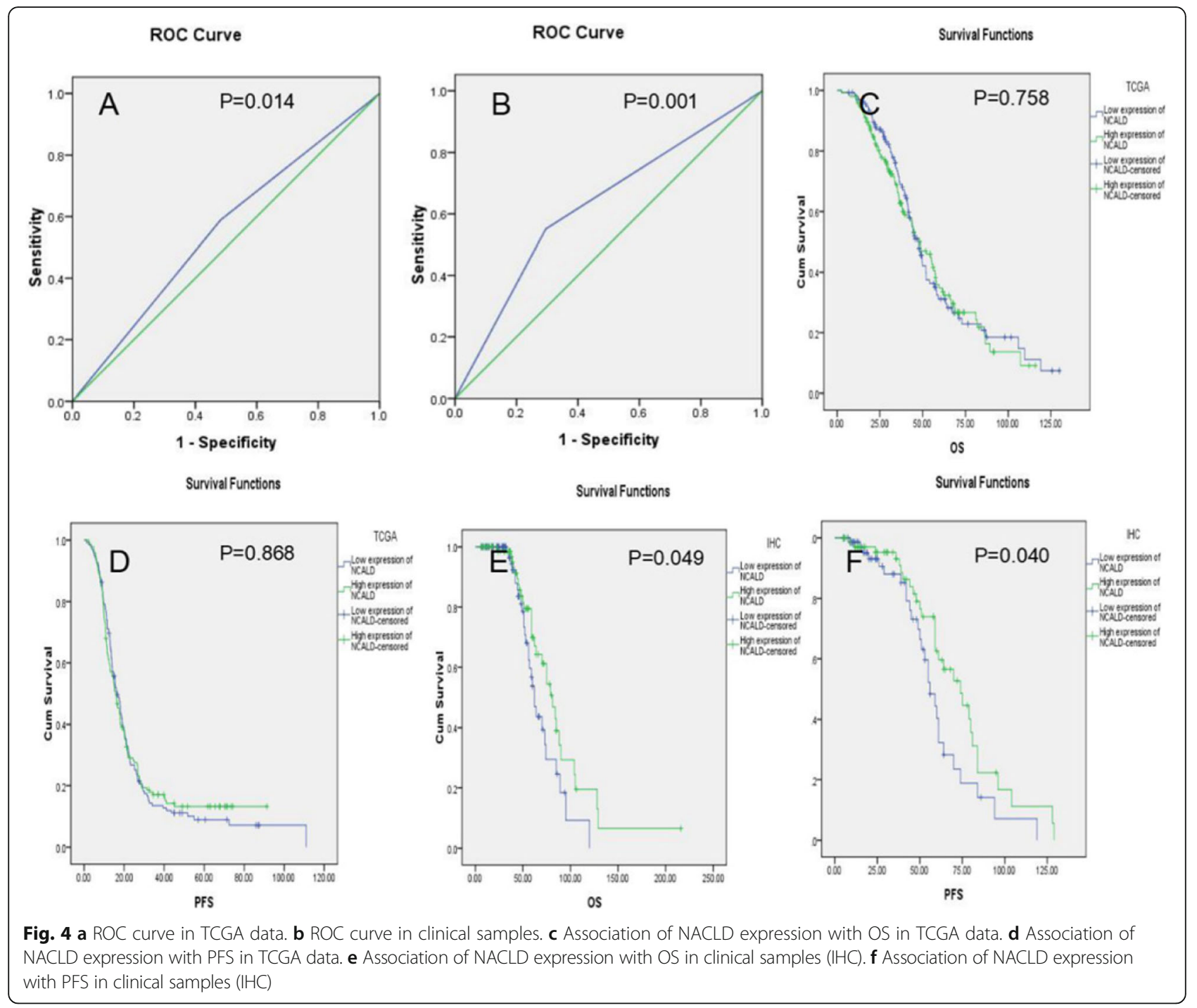

Low expression of NCALD is associated with poor OS and PFS

In the TCGA data, Kaplan-meier survival analysis showed that OS and PFS were shorter in patients with low expression of NCALD, but the differences were not statistically significant (OS:48.72(95\%CI 39.27-58.17) VS 47.37(95\%CI 42.5552.19); PFS:16.59(95\%CI 14.25-18.93) VS $15.77(95 \% \mathrm{CI}$ 13.16-18.38)). In clinical samples, patients with low expression of NCALD were associated with poorer OS and PFS (OS:81.00(95\%CI 68.18-93.82) VS 62.00(95\%CI 55.76-68.24); PFS:74.00(95\%CI $58.32-89.68)$ VS 56.00 (95\%CI $49.02-$ 62.98)). The survival curve is shown in Fig. $4 \mathrm{c}-\mathrm{f}$.

\section{Discussion}

Chemotherapy resistance is the main clinical obstacle in the treatment of ovarian cancer [15]. There is an urgent need to explore new biomarkers that can predict chemotherapy resistance early and therapeutic strategies to reverse resistance. NCALD is a member of the neuronal calcium sensor family. It involves in neuronal diseases and nutritional metabolic diseases. Such as spinal muscular atrophy [16-18], epilepsy [19, 20], depression [21], obesity [22], diabetes [23] and celiac disease [24]. Currently, there is little research on NCALD in ovarian cancer. Our study explored the potential signaling pathways of NCALD and evaluated its ability to predict chemotherapy outcomes and prognosis.

Our GSEA results showed that the genes expression related to ERK1/2 signal pathway, NF-kappaB signaling pathway and immune response pathway were increased in the NCALD overexpression ovarian cancer patients. ERK1 (44 kDa) and ERK2 (42 kDa) are homologous subtypes of ERK family and have the same substrate specificity $[25,26]$. Erk $1 / 2$ is an important component of the cascade of Ras-Raf-MEK-ERK signaling pathway, which has received extensive attention in tumor resistance and tumor therapy [27-29]. It is activated in many cisplatin treated cell lines [30-33]. In the resting state, ERK1/2 
Table 3 The ability of NCALD to predict chemotherapy outcomes in epithelial ovarian cancer

\begin{tabular}{lllllllll}
\hline NCALD expression & AUC & $P$ & Youden & Sensitivity & Specificity & Accuracy & Positive likelihood ratio & Negative likelihood ratio \\
\hline TCGA & $0.59(95 \% \mathrm{Cl} 0.52-0.66)$ & $0.014^{*}$ & 0.16 & 0.70 & 0.46 & 0.53 & 1.28 & 0.67 \\
IHC & $0.64(95 \% \mathrm{Cl} 0.56-0.73)$ & $0.001^{*}$ & 0.27 & 0.59 & 0.68 & 0.61 & 1.82 & 0.61
\end{tabular}

Note: "** means $p<0.05$. Youden $=$ sensitivity + specificity- 1

binds to MEK in the cytoplasm. After phosphorylation, p-ERK $1 / 2$ is transferred to the nucleus and regulates the activity of some transcription factors, such as: c-fos, cJun, Elk-1, STATs, c-myc, NF-jB. Through the abnormal activation of ERK signal, genetic alterations in Ras or RAF family members lead to rapid tumor growth and resistance to apoptosis, which resulting in chemotherapy resistance [34-36]. NF-kB (transcripton factor nuclear kappa B) is an important mediator that acts in the chronic inflammaton [37]. NF-kB was highly expressed in ovarian cancer and related to ovarian cancer progression [38, 39]. Immunotherapies attenuate the levels of nuclear factor kappa B, reduce cell dynamics and effectively target the TLR-related downstream molecules, eliciting a protective effect against chemoresistance [40].

We analyzed the relationship between NCALD expression and chemotherapy outcomes in ovarian cancer patients. First, TCGA data showed that NCALD expression significantly decreased in chemotherapy resistant patients compared to chemotherapy sensitive patients. Second, in local clinical samples, QRT-PCR and immunohistochemical results confirmed that NCALD expression was significantly reduced in chemotherapy resistant patients compared to chemotherapy sensitive patients. Third, stratified analysis showed that in FIGO III-IV stage patients, grade 3 patients and other histology types (except serous and mucinous) patients, NCALD expression in chemotherapy sensitive patients was higher than that in chemotherapy resistant patients. In TCGA data and immunohistochemical samples, the AUC of NCALD expression predicting chemotherapy outcome was 0.59 (95\% CI 0.52-0.66) and 0.64 (95\% CI 0.56-0.73), respectively. NCALD expression has certain diagnostic value for chemosensitivity.

In TCGA data, NCALD expression was not significantly correlated with OS and PFS. But in local clinical samples, patients with low expression of NCALD were associated with poorer OS and PFS, confirming Isaksson's findings. We found that in TCGA and clinical samples, the number of patients with chemotherapy resistance who achieved optimal surgical debulking was significantly less than patients with chemotherapy sensitivity. Residual lesions are associated with the risk of chemoresistance. Rauh-Hain's study showed that residual tumor mass $>1 \mathrm{~cm}$ was a risk factor associated with the risk of platinum resistance recurrence [41]. Luo retrospectively analyzed 341 patients with FIGO III-IV stage epithelial ovarian cancer patients. Postoperative residual tumor mass $>1 \mathrm{~cm}$ was also found to be a risk factor for platinum resistance recurrence $(\mathrm{OR}=2.92 ; 95 \% \mathrm{CI} 1.78-4.77, P=0.000)$ [42]. It can be seen that our results are consistent with research by Rauh-Hain and Luo.

There are several shortcomings in this study. First, bioinformatics results showed that the genes expression related to ERK1/2 signal pathway was increased in the NCALD overexpression ovarian cancer patients. This results need to be verified by confirmatory studies. Second, the ROC curves, while having some predictive value, are not very impressive. Predicting chemotherapy resistance is difficult, especially for single biomarker. It will be necessary to further improve the predictive efficacy by combining with other biomarker.

\section{Conclusions}

In summary, our study demonstrates that NCALD may activate the ERK1 / 2 signaling pathway in ovarian cancer. As a new biomarker of chemotherapy sensitivity, NCALD was significantly down-regulated in chemotherapy resistance ovarian cancer patients. Low expression of NCALD in ovarian cancer is associated with poor OS and PFS. In the future, further research will be needed on the potential mechanism and clinical application value of NCALD in ovarian cancer.

\section{Supplementary information}

Supplementary information accompanies this paper at https://doi.org/10. 1186/s13048-020-00635-6.

Additional file 1: Supplementary document 1. The complete list of differentially expressed genes. Supplementary document $\mathbf{2}$. The complete list of differential gene set. Supplementary document $\mathbf{3}$ Stratified analysis.

\section{Abbreviations}

AUC: Area Under Curve; FIGO: The international federation of gynecology and obstetrics; GSEA: Gene Set Enrichment Analysis; NCALD: Neurocalcin delta; OS: Overall survival; PFS: Progression-free survival; ROC: Receiver Operating Characteristic; TCGA: The Cancer Genome Atlas

\section{Acknowledgements}

Not applicable.

\section{Authors' contributions}

1. First author: Li-yuan Feng, responsible for statistical analysis of data and writing articles. 2. Corresponding author: professor Li li, responsible for the revision. The author(s) read and approved the final manuscript.

\section{Funding}

This work was supported by grants from the National Natural Science Foundation of China (Grant No. 81572579, No. 81660430). The funder had no role in study design, data collection and analysis, decision to publish, or preparation of the manuscript. 


\section{Availability of data and materials}

Not applicable.

\section{Ethics approval and consent to participate}

Approval of ethical review board Number: LW2019034. The research on human genetic resources materials in the "NCALD may induce chemoresistance in epithelial ovarian cancer through ERK1/2 signaling pathway" by Feng Liyuan was received by the Ethics Committee of the affiliated tumor hospital of Guangxi Medical University, and it was considered that the study met the requirements of medical ethics.

\section{Consent for publication}

Not applicable.

\section{Competing interests}

The authors declare that they have no competing interests.

Received: 3 January 2020 Accepted: 12 March 2020

Published online: 30 March 2020

\section{References}

1. Siegel RL, Miller KD, Jemal A. Cancer statistics, 2019. CA Cancer J Clin. 2019; 69(1):7-34.

2. Torre LA, Trabert B, DeSantis CE, et al. Ovarian cancer statistics, 2018. CA Cancer J Clin. 2018;68(4):284-96.

3. Lheureux S, Gourley C, Vergote I, et al. Epithelial ovarian cancer. Lancet. 2019;393(10177):1240-53

4. Norouzi-Barough L, Sarookhani MR, Sharifi M, et al. Molecular mechanisms of drug resistance in ovarian cancer. J Cell Physiol. 2018;233(6):4546-62.

5. Damia G, Broggini M. Platinum Resistance in Ovarian Cancer: Role of DNA Repair. Cancers (Basel). 2019;11(1):119.

6. Monk BJ, Herzog TJ, Tewari KS. Evolution of Chemosensitivity and resistance assays as predictors of clinical outcomes in epithelial ovarian Cancer patients. Curr Pharm Des. 2016;22(30):4717-28.

7. Makar AP, Trope CG, Tummers P, et al. Advanced ovarian Cancer: primary or interval Debulking? Five categories of patients in view of the results of randomized trials and tumor biology: primary Debulking surgery and interval Debulking surgery for advanced ovarian Cancer. Oncologist. 2016; 21(6):745-54.

8. Wang W, Zhou Z, Zhao W, et al. Molecular cloning, mapping and characterization of the human neurocalcin delta gene (NCALD). Biochim Biophys Acta. 2001;1518(1-2):162-7.

9. Viviano J, Krishnan A, Wu H, et al. Data on the calcium-induced mobility shift of myristoylated and non-myristoylated forms of neurocalcin delta. Data Brief. 2016;7:630-3.

10. Hoareau E, Belley N, Klinker K, et al. Characterization of neurocalcin delta membrane binding by biophysical methods. Colloids Surf B: Biointerfaces. 2019;174:291-9.

11. Song $Y$, Zhang $W$, He $X$, et al. High NCALD expression predicts poor prognosis of cytogenetic normal acute myeloid leukemia. J Transl Med. 2019;17(1):166

12. Shi X, Ma C, Zhu Q, et al. Upregulation of long intergenic noncoding RNA 00673 promotes tumor proliferation via LSD1 interaction and repression of NCALD in non-small-cell lung cancer. Oncotarget. 2016;7(18):25558-75.

13. Isaksson HS, Sorbe B, Nilsson TK. Whole genome expression profiling of blood cells in ovarian cancer patients -prognostic impact of the CYP1B1, MTSS1, NCALD, and NOP14. Oncotarget. 2014;5(12):4040-9.

14. Meng $Q$, Duan $P$, Li L, et al. Expression of placenta growth factor is associated with unfavorable prognosis of advanced-stage serous ovarian Cancer. Tohoku J Exp Med. 2018;244(4):291-6.

15. Webb PM, Jordan SJ. Epidemiology of epithelial ovarian cancer. Best Pract Res Clin Obstet Gynaecol. 2017;41:3-14.

16. Torres-Benito L, Schneider S, Rombo R, et al. NCALD antisense oligonucleotide therapy in addition to Nusinersen further ameliorates spinal muscular atrophy in mice. Am J Hum Genet. 2019;105(1):221-30.

17. Riessland M, Kaczmarek A, Schneider S, et al. Neurocalcin Delta suppression protects against spinal muscular atrophy in humans and across species by restoring impaired endocytosis. Am J Hum Genet. 2017:100(2):297-315.

18. Hosseinibarkooie S, Schneider S, Wirth B. Advances in understanding the role of disease-associated proteins in spinal muscular atrophy. Expert Rev Proteomics. 2017;14(7):581-92.
19. Upadhyay A, Hosseinibarkooie S, Schneider S, et al. Neurocalcin Delta knockout impairs adult neurogenesis whereas half reduction is not pathological. Front Mol Neurosci. 2019;12:19.

20. Kuroda Y, Ohashi I, Saito T, et al. Refinement of the deletion in 8q22.2-q22.3 the minimum deletion size at $8 q 22.3$ related to intellectual disability and epilepsy. Am J Med Genet A. 2014;164A(8):2104-8.

21. Lin $\mathrm{E}, \mathrm{Kuo} \mathrm{PH}$, Liu $\mathrm{YL}$, et al. A deep learning approach for predicting antidepressant response in major depression using clinical and genetic biomarkers. Front Psychiatry. 2018;9:290

22. Ma WW, Ding BJ, Yuan LH, et al. Neurocalcin-delta: a potential memoryrelated factor in hippocampus of obese rats induced by high-fat diet. Afr Health Sci. 2017;17(4):1211-21.

23. Kamiyama M, Kobayashi M, Araki $S$, et al. Polymorphisms in the $3^{\prime}$ UTR in the neurocalcin delta gene affect mRNA stability, and confer susceptibility to diabetic nephropathy. Hum Genet. 2007:122(3-4):397-407.

24. Monten C, Gudjonsdottir AH, Browaldh L, et al. Genes involved in muscle contractility and nutrient signaling pathways within celiac disease risk loc show differential mRNA expression. BMC Med Genet. 2015;16:44.

25. Gille $H$, Sharrocks AD, Shaw PE. Phosphorylation of transcription factor p62TCF by MAP kinase stimulates ternary complex formation at c-fos promoter. Nature. 1992;358(6385):414-7.

26. Boulton TG, Yancopoulos GD, Gregory JS, et al. An insulin-stimulated protein kinase similar to yeast kinases involved in cell cycle control. Science. 1990;249(4964):64-7.

27. Cargnello M, Roux PP. Activation and function of the MAPKs and their substrates, the MAPK-activated protein kinases. Microbiol Mol Biol Rev. 2011;75(1):50-83.

28. Fisher R, Larkin J. Vemurafenib: a new treatment for BRAF-V600 mutated advanced melanoma. Cancer Manag Res. 2012;4:243-52.

29. Wada T, Penninger JM. Mitogen-activated protein kinases in apoptosis regulation. Oncogene. 2004;23(16):2838-49.

30. Persons DL, Yazlovitskaya EM, Cui W, et al. Cisplatin-induced activation of mitogen-activated protein kinases in ovarian carcinoma cells: inhibition of extracellular signal-regulated kinase activity increases sensitivity to cisplatin. Clin Cancer Res. 1999:5(5):1007-14.

31. $\mathrm{Xu} \mathrm{H}, \mathrm{Li} Y$, Chen $\mathrm{L}$, et al. SIRT2 mediates multidrug resistance in acute myelogenous leukemia cells via ERK1/2 signaling pathway. Int J Oncol. 2016;48(2):613-23

32. Yang $T, X u F$, Sheng $Y$, et al. A targeted proteomics approach to the quantitative analysis of ERK/BCl-2-mediated anti-apoptosis and multidrug resistance in breast cancer. Anal Bioanal Chem. 2016:408(26): 7491-503.

33. Yokoyama $N$, Matsunobu $T$, Matsumoto $Y$, et al. Activation of ERK1/2 causes Pazopanib resistance via Downregulation of DUSP6 in synovial sarcoma cells. Sci Rep. 2017;7:45332

34. Dilruba S, Michaelis M, Cinatl J Jr, et al. Relevance of subcellular localization of extracellular signal-regulated kinase 1/2 (ERK1/2) for cisplatin resistance. Int J Clin Pharmacol Ther. 2015:53(12):1041-5.

35. Tresini $M$, Lorenzini A, Frisoni $L$, et al. Lack of Elk-1 phosphorylation and dysregulation of the extracellular regulated kinase signaling pathway in senescent human fibroblast. Exp Cell Res. 2001;269(2):287-300.

36. Germann UA, Furey BF, Markland W, et al. Targeting the MAPK signaling pathway in Cancer: promising preclinical activity with the novel selective ERK1/2 inhibitor BVD-523 (Ulixertinib). Mol Cancer Ther. 2017;16(11):2351-63.

37. Chuffa LG, Fioruci-Fontanelli BA, Mendes LO, et al. Melatonin attenuates the TLR4-mediated inflammatory response through MyD88- and TRIFdependent signaling pathways in an in vivo model of ovarian cancer. BMC Cancer. 2015;15:34.

38. Yilmaz E, Gul M, Melekoglu R, et al. Immunhistochemical analysis of nuclear factor kappa Beta expression in etiopathogenesis of ovarian tumors1. Acta Cir Bras. 2018;33(7):641-50.

39. Yang W, Liu L, Li C, et al. TRIM52 plays an oncogenic role in ovarian cancer associated with NF-kB pathway. Cell Death Dis. 2018;9(9):908.

40. Lupi LA, Delella FK, Cucielo MS, et al. P-MAPA and Interleukin-12 Reduce Cell Migration/Invasion and Attenuate the Toll-Like Receptor-Mediated Inflammatory Response in Ovarian Cancer SKOV-3 Cells: A Preliminary Study. Molecules. 2019;25(1). https://doi.org/10.3390/molecules25010005

41. Rauh-Hain JA, Rodriguez N, Growdon WB, et al. Primary debulking surgery versus neoadjuvant chemotherapy in stage IV ovarian cancer. Ann Surg Oncol. 2012:19(3):959-65. 
42. Luo Y, Lee M, Kim HS, et al. Effect of neoadjuvant chemotherapy on platinum resistance in stage IIIC and IV epithelial ovarian cancer. Medicine (Baltimore). 2016;95(36):e4797.

\section{Publisher's Note}

Springer Nature remains neutral with regard to jurisdictional claims in published maps and institutional affiliations.

Ready to submit your research? Choose BMC and benefit from:

- fast, convenient online submission

- thorough peer review by experienced researchers in your field

- rapid publication on acceptance

- support for research data, including large and complex data types

- gold Open Access which fosters wider collaboration and increased citations

- maximum visibility for your research: over $100 \mathrm{M}$ website views per year

At $\mathrm{BMC}$, research is always in progress. 